\title{
The Production of Interleukin-8 Is Increased in Plasma and Peripheral Blood Mononuclear Cells of Patients with Fatigue
}

\author{
Matthew Sorenson ${ }^{1 *}$, Leonard Jason ${ }^{2}$, Athena Lerch ${ }^{2}$, Nicole Porter ${ }^{2}$, Jonna Peterson ${ }^{3}$, \\ Herbert Mathews ${ }^{4}$ \\ ${ }^{1}$ School of Nursing, DePaul University, Chicago, USA; ${ }^{2}$ Department of Psychology, DePaul University, Chicago, USA; ${ }^{3}$ Rush Uni- \\ versity Medical Center, Chicago, USA; ${ }^{4}$ Microbiology and Immunology, Loyola University Chicago, Chicago, USA. \\ Email: *msorenso@depaul.edu
}

Received December $11^{\text {th }}$, 2011; revised January $17^{\text {th }}$, 2012; accepted February $26^{\text {th }}, 2012$

\begin{abstract}
Background and Objective: Previous research has speculated on the role of pro- and inflammatory immune mediators in the etiologic process of fatigue, with contrasting findings. The preponderance of the evidence supports the role of inflammatory mediators in the disease process of Chronic Fatigue Syndrome (CFS). The purpose of this study was to evaluate the production of interleukin IL-8 by peripheral blood mononuclear cells derived from individuals with chronic fatigue. A secondary objective was to determine if there was a significant relationship between IL-8 production and plasma cortisol level. Materials and Methodology: Data was collected from three groups, a sample of individuals with CFS ( $n=15)$, a comparison sample of individuals with fatigue that did not meet criteria for CFS $(n=30)$ and a group of putatively health normative controls $(n=23)$. Peripheral blood was drawn and plasma samples derived. Peripheral blood mononuclear cells were stimulated with a mitogenic protocol and levels of IL-8 in unstimulated and stimulated bulk supernatant determined. Results: Both fatigue groups displayed significantly higher levels of IL-8 than the control group. The CFS group demonstrated higher levels of IL-8 in plasma than the fatigue comparison group. Conclusion: The findings demonstrate a clear association between fatigue and IL-8. The ability to identify an inflammatory marker in association with fatigue could provide a means of identifying those who may be at risk for the development of this disorder.
\end{abstract}

Keywords: Cytokines; Fatigue; Interleukin; PBMC

\section{Introduction}

Estimates propose that Myalgic Encephalomyelitis/Chronic Fatigue Syndrome (ME/CFS) affects approximately 800,000 Americans, at a cost of billions of dollars to the economy [1]. While there have been many studies attempting to outline the immunological correlates of ME/CFS, little has been accomplished by way of establishing a standardized clinical or experimental diagnostic tool for classifying a patient with this disease [2]. Immune abnormalities such as low Natural Killer (NK) cell function, and imbalance in the type I and type II immune response have been suggested, however less investigated are a related group of proteins known as chemokines that are primarily chemoattractant in nature. In other words, this group of signaling proteins stimulates the movement of immune cells in the circulation and contributes to the trafficking of cells into tissue. In an apparently immunologically fa-

\footnotetext{
"Corresponding author.
}

cilitated disease state such as CFS, understanding the role of these proteins is critical.

Chemokines can be broadly classified into two groups; those involved in homeostasis and those associated with inflammation and immune response. These are elicited through infection or injury. One preeminent inflammatory chemokine is CXCL8, also known as IL-8. Interleukin-eight (IL-8) is produced by several epithelial and endothelial cell types, as well as by macrophages and other immune cell classes. The primary role of IL-8 is as chemoattractant with a significant effect on neutrophils. IL-8 also has significant angiogenic properties profoundly influencing the innate immune response [3].

Previous studies have investigated the relationships among pro-inflammatory cytokines and CFS [4-9]. The studies have produced inconsistent findings with some literature supporting an increase in pro-inflammatory mediators while other studies support an increase in anti-inflammatory mediators. Individuals with fibromyalgia, a 
condition characterized by increased fatigue in association with pain, display significantly higher levels of serum IL-8 than controls [10]. Peripheral blood mononuclear cells in those with fibromyalgia have been found to produce higher levels of IL-8 on stimulation than the cells of putatively health controls. The production of IL-8 coincides with marked production of pro-inflammatory proteins such as IL-6 [11]. Furthermore, IL-8 production is associated with pain and is believed to reflect the intimate tie between this protein and inflammation [12,13].

Other lines of investigation have demonstrated CFS and other fatiguing conditions to be associated with viral exposure with an apparent relationship among the release of chemokines and virus infection. Certain viruses are capable of modifying the expression of chemokine receptors, allowing the virus to evade the immune system [14]. A viral homologue of IL-8 utilizes the same signaling pathways as IL-8, providing a selective advantage to the virus in peripheral blood mononuclear cells [15]. Blood derived mast cells excrete several chemokines upon viral exposure, and particularly produce higher levels of IL-8 in response to low levels of virus. In turn, IL-8 appears to stimulate natural killer cells [16]. These findings demonstrate that viral etiologies can influence the production of immunologic variables and potentially contribute to disease pathogenesis.

Previous work has found increased levels of IL-8 in the cerebrospinal fluid of individuals with an apparent viral onset of CFS [7]. Individuals with CFS have been found to display an increased number of apoptotic neutrophils with a concomitant reduction in cell viability in comparison to putatively healthy controls [17]. This finding may reflect an increase in levels of IL-8 in those with CFS. Production of IL-8 may indicate infection with known associated fatigue and with potential dysregulation of the innate immune system in those with CFS.

The association of IL-8 with inflammation can serve to indicate an immune system that has been activated through exposure to infection or other stimuli. In particular, increased levels of IL-8 are anticipated in response to viral or bacterial exposure. CFS has been conceptualized to be associated with viral exposure and immune system dysfunction has been conceptualized as contributing to the pathogenesis of CFS. The relationship between viral etiologies and IL-8, concomitant with the role played by this chemokine in pain and other illness states associated with fatigue make it a candidate for investigation. The ability to identify an inflammatory marker in association with CFS could provide a means of identifying those who may be at risk for the development of this disorder. The purpose of this study was to evaluate the production of IL-8 by peripheral blood mononuclear cells derived from individuals with chronic fatigue. A secondary objective was to determine if there was a significant relationship between IL-8 production and plasma cortisol level.

\section{Method}

\subsection{Participants}

A total of 68 participants (Mean age $=40.5, \mathrm{SD}=13.9$ ) participated in the study. Three separate subgroups were included; 15 participants who met diagnostic criteria for chronic fatigue syndrome, 30 participants that demonstrated chronic fatigue over a period of at least six months with a medical diagnosis other than CFS, and 23 putatively healthy participants served as controls. Participants with chronic fatigue were recruited from a list of individuals who had previously participated in an epidemiological study on CFS. All CFS participants positively identified as meeting criteria for CFS [18] and diagnosed by a physician were considered for inclusion. Individuals that presented with less than four Fukuda symptoms or meeting criteria for medical or psychiatric exclusions were excluded. All persons involved in the research study were at least 18 years of age, not pregnant, able to speak English, and physically and willfully able to travel to the research center.

All patients in this study were screened by a licensed physician. Medical examinations included general neurological and physical assessments, as well as a more in-depth evaluation of medical and neurological history. The evaluation additionally included the administration of several structured clinical instruments including the SF-36 [2]. These measures were intended to assess the symptoms, and medical history, of those with CFS in order to rule out any co-occurring medical conditions. Additional medical information deemed relevant to the study was collected to avoid medical confounds for CFS diagnosis, including exposure to HIV/AIDS, tuberculosis, and other non HIV/ AIDS sexually transmitted diseases. Information on prescribed and illicit drug use was also assessed and recorded. A history of all CFS like symptoms was reported as a final measure. Laboratory tests included a complete blood cell count with differential and platelet count, chemistry screen (which assesses thyroid, renal, and liver functioning), Hepatitis B, Lyme disease screen, erythrocyte sedimentation rate, arthritic profile (which includes rheumatoid factor and anti-nuclear antibody), HIV screen and urinalysis. A tuberculin skin test was also performed. The project physician performed a detailed medical examination to detect evidence of diffuse cardiac or pulmonary dysfunction, adenopathy, synovitis, neuropathy, myopathy, or hepatosplenomegaly. The need for such extensive laboratory tests was exclusionary rather than inclusionary, as defined by Fukuda et al., 2004.

Those excluded from the diagnostic category of those with CFS $(n=15)$ provided a fatigued comparison group, as all participants had reported the presence of fatigue for a period of at least six months. A control group $(n=23)$ 
that was age and gender range (Mean age 43.5, $\mathrm{SD}=$ 2.56) matched to the fatigue sample population was recruited as part of a separate investigation. The control sample was comprised of putatively healthy individuals with no history of significant illness or injury. All control subjects were free of current symptoms of cardiovascular, respiratory, neoplastic or other autoimmune disorders.

\subsection{Peripheral Blood Samples}

Peripheral blood was drawn into lithium citrate tubes and processed within six to eight hours of draw. Blood was processed within six hours of sample procurement. Plasma samples were obtained from blood collection tubes which were spun for 20 minutes at $1000 \times \mathrm{g}$, and plasma aliquots were pipetted directly from each tube with Phosphate Buffered Saline used for volume replacement. Afterward heparinized peripheral blood was overlaid onto Ficoll/Hypaque and then centrifuged at $1000 \times \mathrm{g}$ for 20 min. Cells at the interface were washed with Hank's Balanced Salt Solution (HBSS) and enumerated with a standard hemacytometer. Cytokine profiles were determined by measuring cytokine production from bulk cultured supernatant fluids of stimulated and un-stimulated peripheral blood mononuclear cells (PBMC).

PBMC's $(1 \times 106)$ were cultured in RPMI 1640 plus $10 \%$ fetal bovine serum (supplemented with glutamine, penicillin, streptomycin), with and without a mixture of phytohaemagglutinin (PHA) and phorbol-12-myristate-13acetate (PMA) (PMA @ 20 ng/well; PHA @ 0.05\%/well) in 24 well plates for $48 \mathrm{hr}$ at $37^{\circ} \mathrm{C}$. After $48 \mathrm{hr}$, aliquots of supernatant fluid were collected and stored $\left(-70^{\circ} \mathrm{C}\right)$ until analyzed. This time period and condition was found to be optimal for production of this chemokine. Values were determined using the BioPlex multi-analyte bead suspension array system (BioRad, Hercules, CA) with array kits purchased from BioRad. Plasma cortisol levels were determined using commercially available kits purchased from R \& D Systems (Minneapolis, MN).

\subsection{Data Analysis}

All data were initially screened and analyzed descriptively. Demographic (age, gender) and clinical variables were analyzed to determine possible independent effects of these variables on immune profiles. All data were coded and entered into a spreadsheet workbook created in Microsoft Excel; data were also entered in SPSS Version 16. Data entries were compared between Microsoft Excel and SPSS to ensure correct entry. All data analyses were conducted using SPSS Version 16. The alpha level determined prior to analysis was $\mathrm{p}=0.05$.

Missing IL-8 and cortisol data was not replaced, resulting in variable sample sizes. All data prior to the conduction of the analysis were examined for the presence of outliers. In general, an outlier was considered to be a value that lay outside two standard deviations from the mean. Any such outlying values were excluded from analysis.

For all statistical analyses performed; Levine's test for equality of variance was applied prior to conduction of t-tests for independent samples. To compare the production between, serial t-tests for independent samples were performed with bonferroni correction.

\section{Results}

IL-8 was measured from stimulated and unstimulated PBMC, and values obtained from plasma. Data representing means, ranges, and SEM are provided in Table $\mathbf{1}$ for each of three study groups. The control group demonstrated an approximately eight-fold increase in the production of IL-8 upon stimulation. In CFS subjects, there

Table 1. Stimulated and unstimulated IL-8 levels for each group.

\begin{tabular}{|c|c|c|c|c|c|}
\hline \multicolumn{2}{|c|}{ Fatigue Groups } & \multirow{2}{*}{$\begin{array}{c}\text { Mean } \\
49.65\end{array}$} & \multirow{2}{*}{$\begin{array}{c}\text { Minimum } \\
0.34\end{array}$} & \multirow{2}{*}{$\begin{array}{c}\text { Maximum } \\
288.0\end{array}$} & \multirow{2}{*}{$\begin{array}{l}\text { SEM } \\
21.45\end{array}$} \\
\hline & IL-8 Unstimulated & & & & \\
\hline & IL-8 Stimulated & 517.31 & 10.18 & $2,418.0$ & 171.72 \\
\hline UIJ & Plasma IL-8 & 0.284 & 0.0115 & 1.22 & 0.099 \\
\hline & Cortisol & 76.73 & 41.174 & 162.87 & 18.24 \\
\hline \multirow{4}{*}{ Fatigue Compariso } & IL-8 Unstimulated & 51.26 & 2.686 & 2881.0 & 21.447 \\
\hline & IL-8 Stimulated & 794.192 & 67.335 & 3120.98 & 113.91 \\
\hline & Plasma IL-8 & 0.082 & 0.019 & 0.650 & 0.029 \\
\hline & Cortisol & 71.57 & 15.77 & 348.94 & 10.22 \\
\hline \multirow{3}{*}{ Controls } & IL-8 Unstimulated & 5.83 & 1.54 & 11.80 & 0.675 \\
\hline & IL-8 Stimulated & 37.51 & 3.44 & 315.92 & 12.94 \\
\hline & Plasma IL-8 & 0.04 & 0.016 & 0.114 & 0.023 \\
\hline
\end{tabular}


was an approximately tenfold increase in the production of IL-8 upon stimulation. A visual representation of this change is provided in Figure 1. For those subjects from the fatigued comparison group, there also approximately a tenfold increase in the production of IL-8 upon stimulation.

\subsection{Controls and CFS}

To compare the production of those with CFS and controls, a t-test for independent samples was performed with bonferroni correction. There was a significant difference between the groups in terms of unstimulated $(\mathrm{t}=$ 2.55, $\mathrm{df}=36, \mathrm{p}<0.001)$ and stimulated $(\mathrm{t}=3.59, \mathrm{df}=35$, $\mathrm{p}=0.02$ ) production of IL-8. There was a mean difference of $43.82 \mathrm{ng} / \mathrm{ml}$ between the two groups in relationship to production from unstimulated cells and a mean difference of $479.8 \mathrm{ng} / \mathrm{ml}$ for stimulated cells. This indicates that individuals with chronic fatigue syndrome produce significantly greater levels of IL-8 than putatively healthy controls. As well, those with CFS had a significantly elevated plasma level of IL-8 in comparison to controls $(\mathrm{t}=1.224, \mathrm{p}=0.024)$.

\subsection{Controls and Fatigued Subjects}

There was a significant difference between the control and the fatigue comparison groups in terms of unstimulated $(\mathrm{t}$ $=3.42, \mathrm{df}=51, \mathrm{p}<0.001)$ and stimulated $(\mathrm{t}=5.78, \mathrm{df}=$ $51, p=0.02$ ) levels of IL-8. This indicates that individuals with fatigue produce significant greater levels of IL-8 than healthy controls.

\subsection{Controls and the Fatigued Comparison Group}

Both the unstimulated and stimulated production of IL-8 was higher in the fatigue comparison group than in the CFS subjects. However, these differences were not significant. The plasma concentrations of IL-8 of the CFS subjects $(\mathrm{M}=284 \mathrm{pg} / \mathrm{ml}, \mathrm{SEM}=98.7)$ was significantly higher than the plasma concentration $(\mathrm{M}=94.29 \mathrm{pg} / \mathrm{ml}$, SEM 29.77) of the fatigue comparison group $(\mathrm{t}=2.203$, $\mathrm{df}=37, \mathrm{p}=0.03$ ).

Individuals in the fatigue comparison group displayed a mean cortisol level of $71.57 \mathrm{ng} / \mathrm{ml}$ (Range $=15.77$ to 348.94). Those in the CFS group exhibited a mean cortisol level of $76.73 \mathrm{ng} / \mathrm{ml}$ (Range $=41.17$ to 162.87), which was not significantly different from those in the fatigue comparison group.

\section{Discussion}

The intent of this investigation was to determine whether quantities of IL-8 are differentially produced by the PBMCs of individuals with and without fatigue. The findings

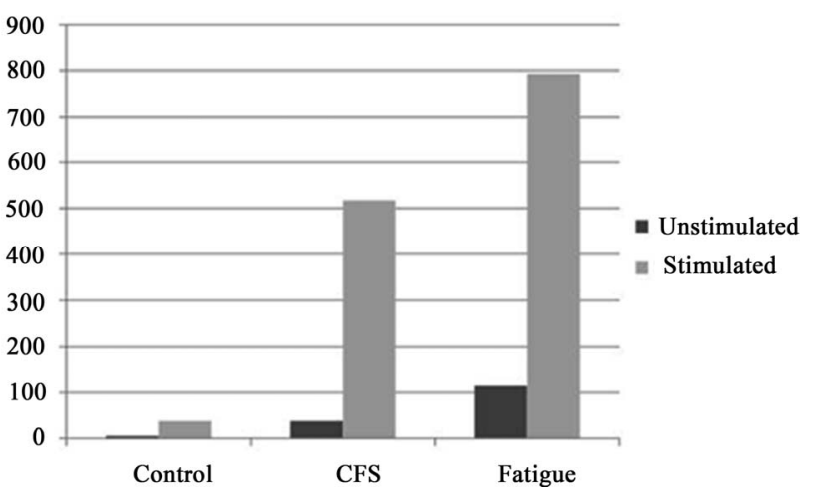

Figure 1. Stimulated and unstimulated IL-8 production groups. Legend: Data presented in $\mathrm{ng} / \mathrm{ml}$, SEM data provided in Table 1.

demonstrate that unstimulated PBMCs of individuals with chronic fatigue produce approximately tenfold the level of IL-8 as do the cells of putatively health controls. When cells were subjected to mitogen stimulation, those with chronic fatigue presented an approximate tenfold increase in the volume of IL-8 secreted, as compared with controls. The amount of IL-8 produced by cells of healthy controls when stimulated was approximately proportional to that produced by the unstimulated cells of those with chronic fatigue. It is then apparent that the PBMC of those with chronic fatigue produce significantly higher levels of IL-8 regardless of the imposition of an immunologic challenge. As well, the plasma concentrations of IL-8 in those with CFS were significantly higher than in a comparison group of fatigued subjects not meeting diagnostic criteria for CFS. This provides a conceptual fit with previous investigations. There are three lines of evidence in CFS populations that help provide a framework in which the production of IL-8 can be considered.

Several authors have speculated that viral exposure and subsequent reactivation may be associated with the development of CFS $[19,20]$. To date, the literature has provided inconsistent support for this concept. It is possible that any viral exposure could trigger a process of inflammation that could eventually result in fatigue. It is also possible that more than one infectious agent needs to be present to evoke a process of inflammation that could result in fatigue. In a murine model, is has been demonstrated that certain bacteria induce the production of IL-8 from the host and appear to utilize the presence of this protein to further infection through the migration of neutrophils [21]. It is also possible that viral exposure could account for the release of IL-8. Individuals exposed to Epstein-Barr Virus have been found to produce higher levels of pro-inflammatory cytokines and IL-8 [22]. Chemokines play a significant role in the recruitment of neutrophils to the site of infection acting as mediators of this response type. The increased presence of IL-8 could represent an underlying inflammatory state in individuals 
with CFS, a state that could be associated with the migration of activated cells into tissue with end effects on endurance and activity. Viral pathologies have been found to be capable of mediating inflammatory pathways through effects on the nuclear factor kappa beta (NFkB) pathway [23], the pathway through which IL-8 is regulated. A possible model then presents itself: CFS may represent a condition in which a genetic predisposition interacts with viral exposure leading to the development of a state of inflammation, a condition which becomes manifest in the development of cognitive deficits and fatigue.

A second line of research into CFS has investigated cognitive deficits and brain lesions associated with this condition. Cytokines and chemokines function within the central nervous system as well as the peripheral providing signaling and mediating inflammation. These proteins contribute to the development of inflammatory lesions in multiple sclerosis and may contribute to the development of brain lesions and cognitive deficits in those with CFS. Individuals with CFS often experience cognitive difficulties with some evidence providing support for the development of CNS lesions [24]. The presence of IL-8 has been found to be associated with mild cognitive impairment and Alzheimer's disease [25]. Chemokine increases could possibly reflect early stages of cognitive deficit, demonstrating a state of reactivity in which activated immune cells are homed to the central nervous system [26]. Additionally characteristic of diseases with a neurodegenerative component is the observed reduction in levels of Brain Derived Neurotrophic Factor (BDNF), a neurotrophin involved in the stimulation of neurogenesis, concomitant with increased levels of proinflammatory chemokines [27]. Glucocorticoid induced reduction in the expression of BDNF associated with chronic stress resulting in both neuronal degeneration and blunted neurogenesis [28-30]. The interaction between BDNF, other immunologic mediators such as IL-8 and glucorticoid regulation may provide further insight into the mechanisms through which hypocortisolism and inflammation interact to produce the neurological and immunological alterations observed in individuals with chronic fatigue. The cognitive deficits seen in those with CFS are likely associated with the brain lesions observed on MRI [24]. The brain lesions found by Lange et al., were predominately areas of white matter hyper-intensity in the frontal lobes. White matter hyperintensities are indicative of tissue loss, often with a vascular etiology [26]. Considering the angiogenic function of IL-8 and its role as a primary pro-inflammatory mediator; it is possible that increased levels of IL- 8 could be one component of a larger process of inflammation and brain lesion development.

The third line of research within which to view IL-8 is the consistent finding of low cortisol levels in individuals with CFS [31-33]. IL-8 is down-regulated by glucocorti- coids and the secretion of IL-8 by TNF $\alpha$ is regulated by the inflammatory transcriptional factor, nuclear factor-Kb (NF-kB) [34]. These considerations may explain the association between hypocortisolism and the proliferation of inflammatory cytokines in association with upregulation of mediators of inflammatory transcription in this population, as the initiation of inflammatory transcription by NF-kB is partially dependent upon reductions in glucocorticoid levels. There was a significant positive correlation found between endogenous cortisol production and unstimulated IL-8 in those with fatigue. This is especially intriguing in light of evidence that individuals with CFS display a dysregulation of the HPA axis. Association and linkage studies have shown a relationship between the genetic components responsible for the regulation of immune and hypothalamic-pituitary-adrenal axis function and CFS [35-37]. Levels of cortisol have been found to be associated with fatigue severity and in the level of pain experienced by individuals with CFS [38]. The absence of an identifiable regulatory element could explain heightened levels of IL-8. The increase in IL-8 could then contribute to inflammatory processes manifesting in fatigue.

No differences were found in this study between individuals diagnosed with CFS and those in the fatigue comparison group, except for significantly higher levels of IL-8 in the plasma of those with CFS. The presence of higher levels of IL-8 in plasma may indicate an active immunologic response in those with CFS.

\section{Conclusion}

This is the first study to examine PBMC production of IL-8 in individuals with chronic fatigue in comparison to a control group, and a fatigued comparison population. Several previous authors have speculated on the role of pro- and inflammatory immune mediators in the etiologic process of fatigue. The finding of significantly increased IL-8 production on the part of PBMC's in this population provides support for immunologic mediation of fatigue and highlights the need for continued examination of immunologic pathways in those with fatigue.

\section{REFERENCES}

[1] L. A. Jason, et al., "A Community-Based Study of Chronic Fatigue Syndrome," Archives of Internal Medicine, Vol. 159, No. 18, 1999, pp. 2129-2137. doi:10.1001/archinte.159.18.2129

[2] L. A. Jason, et al., "Evaluating the Centers for Disease Control's Empirical Chronic Fatigue Syndrome Case Definition,” Journal of Disability Policy Studies, Vol. 20, No. 2, 2009, pp. 93-100. doi:10.1177/1044207308325995

[3] P. Murphy, “Chemokines,” In: W. E. Paul, Ed., Fundamental Immunology, Lippincott Williams \& Wilkins, Philadelphia, 2008. 
[4] J. J. LaManca, et al., "Immunological Response in Chronic Fatigue Syndrome Following a Graded Exercise Test to Exhaustion," Journal of Clinical Immunology, Vol. 19, No. 2, 1999, pp. 135-142. doi:10.1023/A:1020510718013

[5] R. B. Moss, A. Mercandetti and A. Vojdani, "TNF-Alpha and Chronic Fatigue Syndrome," Journal of Clinical Immunology, Vol. 19, No. 5, 1999, pp. 314-316. doi:10.1023/A:1020595709352

[6] B. H. Natelson, M. H. Haghighi and N. M. Ponzio, "Evidence for the Presence of Immune Dysfunction in Chronic Fatigue Syndrome," Clinical and Vaccine Immunology, Vol. 9, No. 4, pp. 747-752.

[7] B. H. Natelson, et al., "Spinal Fluid Abnormalities in Patients with Chronic Fatigue Syndrome," Clinical and Diagnostic Laboratory Immunology, Vol. 12, No. 1, 2005, pp. 52-55.

[8] R. Patarca, "Cytokines and Chronic Fatigue Syndrome," Annals of the New York Academy of Sciences, Vol. 933, 2001, pp. 185-200. doi:1749-6632.2001.tb05824.x

[9] R. Patarca-Montero, et al., "Cytokine and Other Immunologic Markers in Chronic Fatigue Syndrome and Their Relation to Neuropsychological Factors,” Applied Neuropsychology, Vol. 8, No. 1, 2001, pp. 51-64. doi:10.1207/S15324826AN0801 7

[10] H. Wang, et al., "The Role of IL-8 in Patients with Fibromyalgia: A Prospective Longitudinal Study of 6 Months," Clinical Journal of Pain, Vol. 25, No. 1, 2009, pp. 1-4. doi:10.1097/AJP.0b013e31817e13a3

[11] D. J. Wallace, et al., "Cytokines Play an Aetiopathogenetic Role in Fibromyalgia: A Hypothesis and Pilot Study," Rheumatology (Oxford), Vol. 40, No. 7, 2001, pp. 743749. doi:10.1093/rheumatology/40.7.743

[12] N. Kotani, et al., "Cerebrospinal Fluid Interleukin 8 Concentrations and the Subsequent Development of Postherpetic Neuralgia,” American Journal of Medicine, Vol. 116, No. 5, 2004, pp. 318-324. doi:10.1016/j.amjmed.2003.10.027

[13] N. Kotani, et al., "Intrathecal Methylprednisolone for Intractable Postherpetic Neuralgia,” New England Journal of Medicine, Vol. 343, No. 21, 2000, pp. 1514-1519. doi:10.1056/NEJM200011233432102

[14] J. S. Friedland, “Chemokines in Viral Disease,” Research in Virology, Vol. 147, No. 2-3, 1996, pp. 131-138. doi:10.1016/0923-2516(96)80227-5

[15] J. L. Liu, et al., "MEQ and V-IL-8: Cellular Genes in Disguise?” Acta Virologica, Vol. 43, No. 2-3, 1999, pp. 94-101.

[16] S. M. Burke, et al., "Human Mast Cell Activation with Virus-Associated Stimuli Leads to the Selective Chemotaxis of Natural Killer Cells by a CXCL8-Dependent Mechanism," Blood, Vol. 111, No. 12, 2008, pp. 5467-5476. doi:10.1182/blood-2007-10-118547

[17] G. Kennedy, et al., "Increased Neutrophil Apoptosis in Chronic Fatigue Syndrome,” Journal of Clinical Pathology, Vol. 57, No. 8, 2004, pp. 891-893. doi:10.1136/jcp.2003.015511

[18] K. Fukuda, et al., "The Chronic Fatigue Syndrome: A Comprehensive Approach to Its Definition and Study,”
Annals of Internal Medicine, Vol. 121, No. 12, 1994, pp. 953-959.

[19] D. V. Ablashi, et al., "Frequent HHV-6 Reactivation in Multiple Sclerosis (MS) and Chronic Fatigue Syndrome (CFS) Patients,” Journal of Clinical Virology, Vol. 16, No. 3, 2000, pp. 179-191.

doi:10.1016/S1386-6532(99)00079-7

[20] D. Buchwald, et al., "Markers of Inflammation and Immune Activation in Chronic Fatigue and Chronic Fatigue Syndrome," Journal of Rheumatology, Vol. 24, No. 2, 1997, pp. 372-376.

[21] M. Akkoyunlu, et al., "Exploitation of Interleukin-8-Induced Neutrophil Chemotaxis by the Agent of Human Granulocytic Ehrlichiosis," Infection and Immunity, Vol. 69, No. 9, 2001, pp. 5577-5588. doi:10.1128/IAI.69.9.5577-5588.2001

[22] S. C. Klein, et al., "Promotion of IL-8, IL10, TNF Alpha and TNF Beta Production by EBV Infection,” Leukemia Research, Vol. 20, No. 8, 1996, pp. 633-636. doi:10.1016/0145-2126(96)00029-X

[23] J. Hiscott, H. Kwon and P. Genin, "Hostile Takeovers: Viral Appropriation of the NF-kappaB Pathway," Journal of Clinical Investigation, Vol. 107, No. 2, 2001, pp. 143151. doi:10.1172/JCI11918

[24] G. Lange, et al., "Brain MRI Abnormalities Exist in a Subset of Patients with Chronic Fatigue Syndrome," Journal of the Neurological Sciences, Vol. 171, No. 1, 1999, pp. 3-7. doi:10.1016/S0022-510X(99)00243-9

[25] D. Galimberti, et al., "Intrathecal Chemokine Synthesis in Mild Cognitive Impairment and Alzheimer Disease," Archives of Neurology, Vol. 63, No. 4, 2006, pp. 538-543. doi:10.1001/archneur.63.4.538

[26] B. Ovbiagele and J. L. Saver, "Cerebral White Matter Hyperintensities on MRI: Current Concepts and Therapeutic Implications," Cerebrovascular Diseases, Vol. 22, No. 2-3, 2006, pp. 83-90. doi:10.1159/000093235

[27] T. Nagatsu and M. Sawada, "Inflammatory Process in Parkinson's Disease: Role for Cytokines," Current Pharmaceutical Design, Vol. 11, No. 8, 2005, pp. 999-1016. doi:10.2174/1381612053381620

[28] H. Cameron, P. Tanapat and E. Gould, "Adrenal Steroids and N-Methyl-D-Aspartate Receptor Activation Regulate Neurogenesis in the Dentate Gyrus of Adult Rats through a Common Pathway,” Neuroscience, Vol. 82, No. 2, 1998, pp. 349-354. doi:10.1016/S0306-4522(97)00303-5

[29] M. A. Smith, et al., "Stress and Glucocorticoids Affect the Expression of Brain-Derived Neurotrophic Factor and Neurotrophin-3 mRNAs in the Hippocampus," Journal of Neuroscience, Vol. 15, No. 3, 1995, pp. 1768-1777.

[30] S. Saito, et al., "Decreased Synaptic Density in Aged Brains and Its Prevention by Rearing under Enriched Environment as Revealed by Synaptophysin Contents," Journal of Neuroscience Research, Vol. 39, No. 1, 1994. pp. 57-62. doi:10.1002/jnr.490390108

[31] M. A. Demitrack and L. J. Crofford, "Evidence for and Pathophysiologic Implications of Hypothalamic-PituitaryAdrenal Axis Dysregulation in Fibromyalgia and Chronic Fatigue Syndrome," Annals of the New York Academy of 
Sciences, Vol. 840, 1998, pp. 684-697. doi:1749-6632.1998.tb09607.x

[32] M. A. Demitrack, et al., "Evidence for Impaired Activation of the Hypothalamic-Pituitary-Adrenal Axis in Patients with Chronic Fatigue Syndrome," Journal of Clinical Endocrinology \& Metabolism, Vol. 73, No. 6, 1991, pp. 1224-1234. doi:10.1210/jcem-73-6-1224

[33] L. V. Scott, S. Medbak and T. G. Dinan, "Blunted Adrenocorticotropin and Cortisol Responses to CorticotropinReleasing Hormone Stimulation in Chronic Fatigue Syndrome,” Acta Psychiatrica Scandinavica, Vol. 97, No. 6, 1998, pp. 450-457. doi:1600-0447.1998.tb10030.x

[34] S. Vlahopoulos, et al., "Nuclear Factor-KappaB-De- pendent Induction of Interleukin-8 Gene Expression by Tumor Necrosis Factor Alpha: Evidence for an Antioxidant Sensitive Activating Pathway Distinct from Nuclear Translocation,” Blood, Vol. 94, No. 6, 1999, pp. 1878-1889.

[35] H. Fang, et al., "Gene Expression Profile Exploration of a
Large Dataset on Chronic Fatigue Syndrome,” Pharmacogenomics, Vol. 7, No. 3, 2006, pp. 429-440. doi:10.2217/14622416.7.3.429

[36] M. S. Rajeevan, et al., "Glucocorticoid Receptor Polymorphisms and Haplotypes Associated with Chronic Fatigue Syndrome," Genes, Brain and Behavior, Vol. 6, No. 2, 2007, pp. 167-176. doi:1601-183X.2006.00244.X

[37] A. K. Smith, et al., "Polymorphisms in Genes Regulating the HPA Axis Associated with Empirically Delineated Classes of Unexplained Chronic Fatigue,” Pharmacogenomics, Vol. 7, No. 3, 2006, pp. 387-394. doi:10.2217/14622416.7.3.387

[38] S. Torres-Harding, et al., "The Associations between Basal Salivary Cortisol and Illness Symptomatology in Chronic Fatigue Syndrome,” Journal of Applied Biobehavioral Research, Vol. 13, No. 3, 2008, pp. 157-180. doi:1751-9861.2008.00033.x 